\title{
PERSISTENTLY FINITE, PERSISTENTLY ARITHMETIC THEORIES
}

\author{
C. J. ASH AND T. S. MILLAR
}

\begin{abstract}
The main result shows that a certain class of theories, the persistently finite, persistently arithmetic theories, have only arithmetic countable models.
\end{abstract}

Introduction. Complete theories having only finitely many countable models (to within isomorphism) were discussed in [Vaught]. A significant class of these, the persistently finite theories, was introduced and studied in [Benda].

An early question in recursive model theory, posed by Nerode, was whether for a decidable theory having only finitely many countable models, every countable model is decidable. The question was answered negatively by Morley. Other theories with these properties were produced by Lachlan and [Peretyat'kin]. These examples were also persistently finite.

A natural extension of this question is whether, for an arithmetic theory having finitely many countable models, every countable model is arithmetic. This question was answered negatively in [Millar 1] where a decidable theory was produced with finitely many countable models but also having a nonarithmetic type. Again, this example is persistently finite.

A complete theory with only arithmetic complete types will be called persistently arithmetic. We prove here that if a persistently arithmetic theory is persistently finite (defined below), then each of its countable models is arithmetic. This leaves open a number of related questions. For example, fixing $T$ a complete theory with only finitely many countable models:

(1) If $T$ is persistently arithmetic, then is every countable model of $T$ arithmetic?

(2) If $T$ is persistently recursive, then is every countable model of $T$ decidable? In this paper we also show that if the answer to (1) is "no", then any such counterexample must have at least five countable models.

0. Preliminaries. An $n$-ary type in a language $L$ is an arbitrary set of formulas of $L$, each having only the free variables $x_{1}, x_{2}, \ldots, x_{n}$. A complete $n$-ary type of a theory $T$ is a type $\Gamma$ for which $T \cup\left\{\varphi\left(c_{1}, \ldots, c_{n}\right): \varphi\left(x_{1}, \ldots, x_{n}\right) \in \Gamma\right\}$ is a complete consistent theory in $L\left(c_{1}, \ldots, c_{n}\right)$. A (complete) type is a (complete) $n$-ary type for some $n$. A persistently finite theory is a complete consistent theory $T$ such that for every complete type $\Gamma\left(x_{1}, \ldots, x_{n}\right)$ of $T$, the theory $\Gamma\left(c_{1}, \ldots, c_{n}\right)$ has only finitely many countable models up to isomorphism.

Where relevant, we assume that the languages considered are effective, that is, the symbols are Gödel-numbered in such a way that the nature of each symbol can be recursively discovered from its Gödel number. The formulas are then numbered in some effective way. A theory or type is arithmetic if the set of Gödel numbers of

Received by the editors July 30, 1982.

1980 Mathematics Subject Classification. Primary 03C57; Secondary 03C50.

Key words and phrases. Decidable models, complete types, hyperarithmetic, undecidable models. 
its members is arithmetic. A persistently arithmetic theory is a complete consistent theory all of whose complete types are arithmetic.

Let $\mathfrak{A}$ be a realization of an effective language $L$ where the domain of $\mathfrak{A}$ is a recursive set of natural numbers. The augmented language $L(\mathfrak{A})$, with constants naming the elements, may then be taken to be effective. For any set $\Phi$ of formulas of $L$, the $\Phi$-diagram of $\mathfrak{A}$ denotes the set of all sentences $\varphi\left(a_{1}, \ldots, a_{n}\right)$ of $L(\mathfrak{A})$ true in $\left(\mathfrak{A},\{a\}_{a \in \mathfrak{A}}\right)$ for which $\varphi\left(x_{1}, \ldots, x_{n}\right) \in \Phi$. The complete diagram of $\mathfrak{A}$ is the $\Phi$ diagram where $\Phi$ is the set of all formulas of $L$. We say that a structure is decidable (arithmetic) if it is isomorphic to one with a recursive (arithmetic) diagram. A straightforward construction establishes the well-known

LEMMA 0. Every consistent arithmetic theory has an arithmetic model.

The language $L^{*}$. In discussing persistently finite theories, it seems useful to introduce the following first-order language, which has the effect of an infinitary language.

Let $\left\{\Gamma_{i}\right\}_{i<\omega}$ be any sequence of types (not necessarily complete) in $L$. Let $L^{*}$ be the result of adding to $L$ new relation symbols $\left\{P_{i}\right\}_{i<\omega}$ where, if $\Gamma_{i}$ is an $n$-ary type then $P_{i}$ is an $n$-ary relation symbol.

For any realization $\mathfrak{A}$ of $L^{\prime} \supseteq L$, let $P_{i}^{\mathfrak{A}}$ be the relations of $\mathfrak{A}$ defined by

$$
P_{i}^{\mathfrak{A}}\left(a_{1}, \ldots, a_{n}\right) \Leftrightarrow \text { for all } \varphi \in \Gamma_{i}, \mathfrak{A} \vDash \varphi\left[a_{1}, \ldots, a_{n}\right] .
$$

We define $\mathfrak{A}^{*}$ to be $\left(\mathfrak{A}, P_{i}^{\mathfrak{A}}\right)_{i<\omega}$ so that $\mathfrak{A}^{*}$ is a realization of $\left(L^{\prime}\right)^{*}$.

We classify the formulas of $\left(L^{\prime}\right)^{*}$ using classes $\Sigma_{n}^{L^{\prime}}$ and $\Pi_{n}^{L^{\prime}}$ for $n<\omega$, defined as follows. Let $\Delta_{0}^{L^{\prime}}$ be the class of Boolean combinations of atomic formulas of $\left(L^{\prime}\right)^{*}$ and arbitrary formulas of $L^{\prime}$. Let $\Sigma_{0}^{L^{\prime}}=\Pi_{0}^{L^{\prime}}=\Delta_{0}^{L^{\prime}}$. Now let $\Sigma_{n+1}^{L^{\prime}}$ be the set of all formulas of the form $\exists y_{1} \exists y_{2} \cdots \exists y_{k} \varphi$ for $k \geq 0$ and $\varphi \in \Pi_{n}^{L^{\prime}}$, and let $\Pi_{n+1}$ be those of the form $\forall y_{1} \forall y_{2} \cdots \forall y_{k} \varphi$ with $k \geq 0$ and $\varphi \in \Sigma_{n}^{L}$.

Note that if $L^{\prime}=L \cup\left\{a_{\alpha} \mid \alpha<\beta\right\}$, where the $a_{i}$ 's are constant symbols, and $\varphi\left(a_{1}, \ldots, a_{s}, y_{1}, \ldots, y_{n}\right) \in\left(L^{\prime}\right)^{*}$, then

$$
\varphi\left(a_{1}, \ldots, a_{s}, y_{1}, \ldots, y_{m}\right) \in \Sigma_{n}^{L^{\prime}}\left(\Pi_{n}^{L^{\prime}}\right) \text { iff } \varphi\left(x_{1}, \ldots, x_{s}, y_{1}, \ldots, y_{m}\right) \in \Sigma_{n}^{L}\left(\Pi_{n}^{L}\right) .
$$

We define $A \equiv_{n}^{*} \mathfrak{B}$ if $A^{*}$ and $\mathfrak{B}^{*}$ satisfy the same $\Sigma_{n}^{L^{\prime}}$ (and also $\Pi_{n}^{L^{\prime}}$ ) sentences, where $A$ and $\mathfrak{B}$ are $L^{\prime}$-structures, $L \subseteq L^{\prime}$. Thus $A \equiv_{0}^{*} \mathfrak{B}$ if and only if $A \equiv \mathfrak{B}$. We also define $\mathfrak{A} \equiv^{*} \mathfrak{B}$ if $\mathfrak{A} \equiv_{n}^{*} \mathfrak{B}$ for each $n<\omega$. Since every formula of $\left(L^{\prime}\right)^{*}$ is logically equivalent to one in some $\Sigma_{n}^{L^{\prime}}$, we have $\mathfrak{A} \equiv^{*} \mathfrak{B}$ if and only if $\mathfrak{A}^{*} \equiv \mathfrak{B}^{*}$.

The language $L^{*}$ has exactly the expressive power of the fragment of $L_{\omega_{1}, \omega}$ obtained from the atomic formulas and the infinite conjunctions $M \Gamma_{i}$ by applying the quantifiers and the finitary connectives.

Notation. The symbol $\bar{a}$ denotes a finite sequence of the form $\left\langle a_{1}, a_{2}, \ldots, a_{m}\right\rangle$ where $m \geq 0$ is either arbitrary or indicated by the context. In $\S 2$, we construct a model in the stype of [Keisler], where more detail is given.

We use the notation $\varphi$ ᄀ for the result of peforming a single step in moving a negation inward. More precisely, $\varphi\urcorner$ is defined to be $\neg \varphi$ if $\varphi$ is atomic; $\psi$ if $\varphi$ is $\neg \psi ; \neg \vartheta \vee \neg \psi$ if $\varphi$ is $\vartheta \& \psi ; \neg \vartheta \& \neg \psi$ if $\varphi$ is $\vartheta \vee \psi ; \forall x \neg \psi$ if $\varphi$ is $\exists x \psi$; $\exists x \neg \psi$ if $\varphi$ is $\forall x \psi$.

1. Characterisation of the models. Let $T$ be a persistently finite theory in the language $L$. Since $T$ has only finitely many countable models, $T$ has only countably 
many complete types. Let $\left\{\Gamma_{i}\right\}_{i<\omega}$ be an enumeration of all the complete types of $T$ in $L$.

LEMMA 1. There exists $n<\omega$ such that for all countable $\mathfrak{A}, \mathfrak{B} \vDash T, \mathfrak{A} \equiv_{n}^{*} \mathfrak{B} \Leftrightarrow$ $\mathfrak{A} \cong \mathfrak{B}$.

Proof. We show this in four steps. First we show:

(1) If $\mathfrak{A}, \mathfrak{B} \vDash T, \bar{a}, a^{\prime} \in \mathfrak{A}, \bar{b} \in \mathfrak{B}$ and if $(\mathfrak{A}, \bar{a}) \equiv_{n+1}^{*}(\mathfrak{B}, \bar{b})$ then there exists $b^{\prime} \in \mathfrak{B}$ for which $\left(\mathfrak{A}, \bar{a}, a^{\prime}\right) \equiv_{n}^{*}\left(\mathfrak{B}, \bar{b}, b^{\prime}\right)$.

Let $\varphi_{1}(x), \varphi_{2}(x), \ldots$ be all the $\Sigma_{n} \cup \Pi_{n}$ formulas satisfied in $(\mathfrak{A}, \bar{a})^{*}$ by $a^{\prime}$. Then for each $k \exists x\left(\varphi_{1}(x) \& \cdots \& \varphi_{k}(x)\right)$ is logically equivalent to a $\Sigma_{n+1}$ sentence true in $(\mathfrak{A}, \bar{a})^{*}$ and is thus true in $(\mathfrak{B}, \bar{b})^{*}$. So there exists $b_{k}^{\prime}$ which satisfies each of $\varphi_{1}(x), \ldots, \varphi_{k}(x)$ in $(\mathfrak{B}, \bar{b})^{*}$.

But one of the $\varphi_{k}(x)$ is of the form $P_{i}(\bar{c}, x)$ which specifies the type realized in $\mathfrak{A}$ by $\bar{a}, a^{\prime}$. So, since $T$ is persistently finite, only finitely many of the $\left(\mathfrak{B}, \bar{b}, b_{k}^{\prime}\right)$ are nonisomorphic. Thus, one of the $b_{k}^{\prime}$ satisfies all of the $\varphi_{i}(x)$. Taking $b^{\prime}$ to be such, we have $\left(\mathfrak{A}, \bar{a}, a^{\prime}\right) \equiv_{n}^{*}\left(\mathfrak{B}, \bar{b}, b^{\prime}\right)$.

We can now show:

(2) If $\mathfrak{A}, \mathfrak{B} \vDash T, \bar{a}, a^{\prime} \in \mathfrak{A}, \bar{b} \in \mathfrak{B}$ and if $(\mathfrak{A}, \bar{a}) \equiv^{*}(\mathfrak{B}, \bar{b})$ then there exists $b^{\prime} \in \mathfrak{B}$ for which $\left(\mathfrak{A}, \bar{a}, a^{\prime}\right) \equiv^{*}\left(\mathfrak{B}, \bar{b}, b^{\prime}\right)$.

From (1), for each $n$, there exists $b_{n}^{\prime}$ for which $\left(\mathfrak{A}, \bar{a}, a^{\prime}\right) \equiv_{n}^{*}\left(\mathfrak{B}, \bar{b}, b_{n}^{\prime}\right)$. Again only finitely many of the $\left(\mathfrak{B}, \bar{b}, b_{n}^{\prime}\right)$ are nonisomorphic, so we may take $b^{\prime}$ to be some $b_{n}^{\prime}$ such that $\left(\mathfrak{A}, \bar{a}, a^{\prime}\right) \equiv_{m}^{*}\left(\mathfrak{B}, \bar{b}, b_{n}^{\prime}\right)$ for infinitely many and thus every $m$.

It now follows that:

(3) If $\mathfrak{A}, \mathfrak{B}$ are countable models of $T$ and if $\mathfrak{A} \equiv \equiv^{*} \mathfrak{B}$ then $\mathfrak{A} \cong \mathfrak{B}$.

Using (2), this is just a routine back-and-forth argument.

Now $T$ has only finitely many nonisomorphic countable models, say $\mathfrak{A}_{1}, \mathfrak{A}_{2}, \ldots$, $\mathfrak{A}_{k}$. By (3), for $i \neq j$, there exists $n_{i j}$ with $\mathfrak{A}_{i} \not_{n_{i j}}^{*} \mathfrak{B}_{j}$. Taking $n$ to be the maximum of the $n_{i j}$ for $1 \leq i<j \leq k$, gives the desired result.

2. Construction of an arithmetic model. We return to the general situation where $T$ is an arithmetic theory in a language $L$ and the $\Gamma_{i}$ are arbitrary types in $L$, not necessarily complete. We assume that the $\Gamma_{i}$ can be listed in such a way that the relation $\varphi \in \Gamma_{i}$ is an arithmetic relation between $i$ and $\varphi$.

LEMMA 2. Let $\varphi_{0}$ be $a \Pi_{n+1}$ sentence of $L^{*}$ such that $T$ has models $\mathfrak{A}$ and $\mathfrak{B}$ for which $\mathfrak{A}$ is arithmetic, $\mathfrak{B}^{*} \vDash \varphi_{0}$ and $\mathfrak{A} \equiv_{n}^{*} \mathfrak{B}$. Then $T$ has an arithmetic model $\mathfrak{B}$, for which $\mathfrak{B}_{1}^{*} \vDash \varphi_{0}$.

Proof. Let $L(C)$ be the language $L$ augmented by a countable set $C=\left\{c_{0}, c_{1}, \ldots\right\}$ of new constant symbols. Let $S$ be a family of finite sets of $\Sigma_{n}$ sentences of $L(C)^{*}$ which are simultaneously satisfiable in $\mathfrak{A}^{*}$. That is, $s \in S$ if $s$ is of the form $\left\{\varphi_{1}(\bar{c}), \ldots, \varphi_{k}(\bar{c})\right\}$ where the $\varphi_{i}(\bar{x})$ are $\Sigma_{n}$ formulas of $L^{*}$ and $\mathfrak{A}^{*} \vDash \exists \bar{x}\left(\varphi_{1}(\bar{x}) \& \cdots \& \varphi_{k}(\bar{x})\right)$

We construct the diagram of $\mathfrak{B}_{1}$ by forming, recursively relative to the $\Sigma_{n^{-}}$ diagram of $\mathfrak{A}^{*}$ and the relation $\varphi \in \Gamma_{i}$, a sequence

$$
s_{0} \subseteq s_{1} \subseteq s_{2} \subseteq \cdots \text { of members of } S
$$

in such a way that $s_{\omega}=\bigcup_{i<\omega} s_{i}$ satisfies each of the following conditions.

(1) If $\varphi \& \psi \in s_{\omega}$ then $\varphi \in s_{\omega}$ and $\psi \in s_{\omega}$.

(2) If $\varphi \vee \psi \in s_{\omega}$ then either $\varphi \in s_{\omega}$ or $\psi \in s_{\omega}$. 
(3) If $\forall x \varphi(x) \in s_{\omega}$ then $\varphi(c) \in s_{\omega}$ for every $c \in C$.

(4) If $\exists x \varphi(x) \in s_{\omega}$ then $\varphi(c) \in s_{\omega}$ for some $c \in C$.

(5) If $\neg \varphi \in s_{\omega}$ then $\varphi \neg \in s_{\omega}$.

(6) If $P_{i}(\bar{c}) \in s_{\omega}$ then $\varphi(\bar{c}) \in s_{\omega}$ for every $\varphi(\bar{x}) \in \Gamma_{i}$.

(7) If $\neg P_{i}(\bar{c}) \in s_{\omega}$ then $\neg \varphi(\bar{c}) \in s_{\omega}$ for some $\varphi(\bar{x}) \in \Gamma_{i}$.

(8) For every sentence $\varphi$ of $L(C)$, either $\varphi \in s_{\omega}$ or $\neg \varphi \in s_{\omega}$.

Suppose that $\varphi_{0}$ is $\forall \bar{x} \psi_{0}(\bar{x})$ where $\psi_{0}(\bar{x})$ is a $\Sigma_{n}$ formula of $L^{*}$.

(9) For every $\bar{c} \in C, \psi_{0}(\bar{c}) \in s_{\omega}$.

To show that this is possible (without yet considering effectiveness) we must, for example considering condition (2), show that if $s \in S$ and $\varphi \vee \psi \in s$ then either $s \cup\{\varphi\} \in S$ or $s \cup\{\psi\} \in S$. By definition of $S, s$ is satisfiable in $\mathfrak{A}^{*}$ by some elements of $\mathfrak{A}$. Thus, either $s \cup\{\psi\}$ or $s \cup\{\varphi\}$ is satisfiable in $\mathfrak{A}^{*}$ by the same element of $\mathfrak{A}$. Each of conditions (1)-(8) has a similar straightforward verification.

For condition (9) we must show that, if $s \in S$ and $\bar{c} \in C$, then $s \cup\left\{\psi_{0}(\bar{c})\right\} \in S$. The conjuction of $s$ is logically equivalent to some $\Sigma_{n}$ sentence $\sigma(\bar{c}, \bar{d})$ where $\sigma(\bar{x}, \bar{y})$ is a $\Sigma_{n}$ formula of $L^{*}$. By definition of $S$, the $\Sigma_{n}$ sentence $\exists \bar{x} \exists \bar{y} \sigma(\bar{x}, \bar{y})$ is true in $\mathfrak{A}^{*}$. Since $\mathfrak{A} \equiv_{n}^{*} \mathfrak{B}, \mathfrak{B}^{*} \vDash \exists \bar{x} \exists \bar{y} \sigma(\bar{x}, \bar{y})$. Since $\mathfrak{B}^{*} \vDash \forall \bar{x} \psi_{0}(\bar{x}), \mathfrak{B}^{*} \vDash \exists \bar{x} \exists \bar{y}\left(\sigma(\bar{x}, \bar{y}) \& \psi_{0}(\bar{x})\right)$. Now since this last sentence is logically equivalent to a $\Sigma_{n}$ sentence and since $\mathfrak{A} \equiv_{n}^{*}$ $\mathfrak{B}$, we have $\mathfrak{A}^{*} \vDash \exists \bar{x} \exists \bar{y}(\sigma(\bar{x}, \bar{y}) \& \psi(\bar{x}))$. Thus $s \cup\left\{\psi_{0}(\bar{c})\right\} \in S$.

Now let $s_{\omega}$ have the properties (1)-(9). We may define $\mathfrak{B}_{1}$ in the usual way by first defining an equivalence relation on $C$ by $c_{i} \sim c_{j}$ if $\left(c_{i}=c_{j}\right) \in s_{\omega}$. Denoting the equivalence class of $c \in C$ by $\langle c\rangle$, we let the domain of $\mathfrak{B}_{1}$ be $\{\langle c\rangle: c \in C\}$ and define the relations, etc., of $\mathfrak{B}_{1}$ by $\mathfrak{B}_{1} \vDash \varphi\left[\left\langle c_{1}\right\rangle, \cdots,\left\langle c_{m}\right\rangle\right]$ iff $\varphi\left(c_{1}, \ldots, c_{m}\right) \in s_{\omega}$ when $\varphi$ is an atomic formula of $L$. These notions can be shown to be well defined using conditions (8), (3) and (2) and the definition of $S$.

We may now prove that if $\varphi\left(c_{1}, \ldots, c_{m}\right) \in s_{\omega}$ then $\mathfrak{B}^{*} \vDash \varphi\left[\left\langle c_{1}\right\rangle, \ldots,\left\langle c_{m}\right\rangle\right]$, first for $\varphi$ a formula of $L$, by induction using conditions (1)-(5), then for $\varphi$ an atomic or negated atomic formula of $L^{*}$, using conditions (6) and (7), and so using (1)-(5) again, for every formula $\varphi$ of $L^{*}$.

So by condition (8) and the definiton of $s, \mathfrak{B}_{1} \vDash T$, and by (9), $\mathfrak{B}_{1} \vDash \varphi_{0}$.

To complete the proof, we must see that the sequence $s_{0} \subseteq s_{1} \subseteq s_{2} \subseteq$. can be chosen to be arithmetic. It follows, by condition (8) that $\mathfrak{B}_{1}$ is arithmetic.

Since $\mathfrak{A}$ is arithmetic, we may assume that the diagram of $\mathfrak{A}$ is arithmetic. Since the relation $\varphi \in \Gamma_{i}$ is arithmetic, the $\Delta_{0}$-diagram of $\mathfrak{A}^{*}$ is arithmetic. Thus, by induction on $k$, the $\Sigma_{k}$-diagram of $\mathfrak{A}^{*}$ is arithmetic for each $k$. Now the sequence $\left\{s_{i}\right\}$ can be constructed arithmetically relative to the $\Sigma_{n}$-diagram of $\mathfrak{A}^{*}$, since each $s_{n+1}$ is obtained from $s_{n}$ by finding a $\Sigma_{n}$ sentence $\varphi$ from some arithmetic set for which $s_{n} \cup\{\varphi\}$ is satisfiable in $\mathfrak{A}^{*}$.

3. Persistently finite, persistently arithmetic theories. These two lemmas now apply to give

THEOREM 1. Let $T$ be a persistently finite persistently arithmetic theory. Then all countable models of $T$ are arithmetic.

Proof. Let $\left\{\Gamma_{i}\right\}_{i<\omega}$ be a list of all complete types of $T$. Since $T$ has finitely many countable models, there is obviously [Benda] a single complete type $\Gamma$ of $T$ such that every model of $T$ realizing $\Gamma$ realizes every type of $T$. Since $T$ is persistently arithmetic, $\Gamma$ is arithmetic. Thus, by Lemma $0, T$ has an arithmetic 
model realizing all types of $T$. So the list $\left\{\Gamma_{i}\right\}$ can be chosen so that the relation $\varphi \in \Gamma_{i}$ is arithmetic.

Now suppose that $\mathfrak{B}$ is a countable model of $T$ which is not arithmetic. (This will eventually be contradicted.) For each nonarithmetic model $\mathfrak{B}$ of $T$, there is by Lemma 1 a sentence of $L^{*}$ true in $\mathfrak{B}^{*}$ but not in any arithmetic model of $T$. Similarly, since $T$ is persistently finite and persistently arithmetic, for each $\bar{b} \in$ $|\mathfrak{B}|^{<\omega}$, there is a single sentence of $L(\bar{b})^{*}$ true in $(\mathfrak{B}, \bar{b})$ but not in any arithmetic model of the arithmetic theory $\operatorname{Th}(\langle\mathfrak{B}, \bar{b}\rangle)$. So for each $\bar{b} \in|\mathfrak{B}|^{<\omega}$, we may let $n$ be the least natural number such that $(\mathfrak{B}, \bar{b})^{*}$ satisfies some $\Pi_{n+1}$ sentence $\varphi_{0}$ of $L(\bar{c})^{*}$ which is not true in $(\mathfrak{A}, \bar{a})^{*}$ for any arithmetic model $(\mathfrak{A}, \bar{a})$ of $\operatorname{Th}(\mathfrak{B}, \bar{b})$. Let $\bar{b}$ be chosen so that this $n$ is least.

For this choice of $\bar{b}$, we may let $\left(\mathfrak{A}_{1}, \bar{a}_{1}\right), \ldots,\left(\mathfrak{A}_{k}, \bar{a}_{k}\right)$ be all the nonisomorphic arithmetic models of $\operatorname{Th}(\mathfrak{B}, \bar{b})$, since $T$ is persistently finite. We now see that if $\varphi$ is any $\Sigma_{n+1}$ sentence of $L(\bar{c})^{*}$ true in $(\mathfrak{B}, \bar{b})^{*}$ then $\varphi$ is true in some $\left(\mathfrak{A}_{1}, \bar{a}_{i}\right)^{*}$. For suppose otherwise and let $\varphi$ be $\exists \bar{x} \psi(\bar{c}, \bar{x})$, where $\psi$ is $\Pi_{n}$. Then there exist $\bar{b}^{\prime} \in \mathfrak{B}$ for which $\psi(\bar{c}, \bar{d})$ is true in $\left(\mathfrak{B}, \bar{b}, \bar{b}^{\prime}\right)^{*}$ but not in $\left(\mathfrak{A}, \bar{a}, \bar{a}^{\prime}\right)^{*}$ for any arithmetic model $\left(\mathfrak{A}, \bar{a}, \bar{a}^{\prime}\right)$ of $\operatorname{Th}\left(\mathfrak{B}, \bar{b}, \bar{b}^{\prime}\right)$. If $n \neq 0$ this contradicts the choice of $\bar{b}$. If $n=0$ then $\psi$ is $\Delta_{0}$, in which case any arithmetic model of $\operatorname{Th}\left(\mathfrak{B}, \bar{b}, \bar{b}^{\prime}\right)$, which exists by Lemma 0 and the fact that $T$ is persistently arithmetic, will satisfy $\psi(\bar{c}, \bar{d})$.

Now let $\varphi_{1}, \varphi_{2}, \ldots$ be all the $\Sigma_{n} \cup \Pi_{n}$ sentences of $L(\bar{c})^{*}$ true in $(\mathfrak{B}, \bar{b})^{*}$. Each of $\varphi_{1} \& \varphi_{2} \& \cdots \& \varphi_{k}$ is equivalent to a single $\Sigma_{n+1}$ sentence and hence is true in some $\left(\mathfrak{A}_{i}, \bar{a}_{i}\right)^{*}$. Thus there is a single $\left(\mathfrak{A}_{i}, \bar{a}_{i}\right)^{*}$ which satisfies all the $\varphi_{k}$. For this $i$, we have $\left(\mathfrak{A}_{i}, \bar{a}_{i}\right) \equiv_{n}^{*}(\mathfrak{B}, \bar{b})$. We may thus apply Lemma 2 to obtain an arithmetic model $\left(\mathfrak{B}_{1}, \bar{b}_{1}\right)$ of $\operatorname{Th}(\mathfrak{B}, \bar{b})$ for which $\left(\mathfrak{B}_{1}, \bar{b}_{1}\right)^{*} \vDash \varphi_{0}$. But this contradicts the choice of $\varphi_{0}$.

4. The number of models. If $T$ is an arithmetic, persistently finite theory which is not persistently arithmetic, then its countable models may not all be arithmetic. But we can then say something about the number of models of $T$. First we will need a lemma [Millar 2].

LEMMA. If for a complete decidable theory there is an r.e. list of all its recursive complete types, and the theory has only countably many complete types, then the theory has a decidable homogeneous model realizing all of its recursive types.

THEOREM 2. If an arithmetic, persistently finite theory $T$ has a countable nonarithmetic model then it has at least 5 countable models.

The proof of the lemma directly relativizes to the arithmetic case, i.e. the lemma is still true if all occurrences of "decidable", "r.e.", and "recursive" are replaced by "arithmetic". The theory $T$ satisfies all of the hypotheses of the relativized lemma. The only part that is perhaps not obvious is whether $T$ must have an arithmetic list of its arithmetic types, but this was argued in $\S 3$. Thus $T$ has a homogenous arithmetic model $\mathfrak{A}_{1}$ realizing all the arithmetic types of $T$.

Next we claim that $T$ must have an arithmetic nonprincipal type. Since all the principal types of an arithmetic theory are arithmetic, and because $T$ is not persistently arithmetic, $T$ must have a nonprincipal $n$-type for some $n$. Fixing such an $n$, define

$$
\begin{aligned}
B\left(x_{1}, \ldots, x_{n}\right) & \\
& =\left\{\neg \varphi\left(x_{1}, \ldots, x_{n}\right) \mid \forall \theta\left(x_{1}, \ldots, x_{n}\right) \exists k T \vdash \varphi\left(x_{1}, \ldots, x_{n}\right) \rightarrow \theta\left(x_{1}, \ldots, x_{n}\right)^{k}\right\} .
\end{aligned}
$$


Thus $B$ is just the collection of negations of formulas in the displayed variables that are either inconsistent with or a complete formula of $T$. Since $T$ has a nonprincipal $n$-type, $T \cup B\left(x_{1}, \ldots, x_{n}\right)$ must be consistent. Since $T$ is arithmetic and thus too $B\left(x_{1}, \ldots, x_{n}\right)$, and since $T$ has only countably many complete types, $T$ has an arithmetic complete type $\Gamma\left(x_{1}, \ldots, x_{n}\right)$ satisfying

$$
B\left(x_{1}, \ldots, x_{n}\right) \subseteq \Gamma\left(x_{1}, \ldots, x_{n}\right) .
$$

This is the desired type, since $\Gamma$ cannot be principal, given its containment of $B$.

Again just as in $\S 3$, there is some arithmetic type of $T$ such that if a model realizes that type then it realizes every arithmetic type of $T$. Since $T$ has a nonprincipal arithmetic type, such a type must also be nonprincipal. So fix $\Gamma\left(x_{1}, \ldots, x_{n}\right)$ arithmetic, nonprincipal and such that any model realizing $\Gamma$ realizes every arithmetic type of $T$.

By the same argument, but relative to the theory $\Gamma(\bar{c})$ instead of $T$, there is an arithmetic type $\Sigma(\bar{x}, \bar{y})$ such that $\Sigma(\bar{c}, \bar{y})$ is a nonprincipal type of the theory $\Gamma(\bar{c})$. Now, since $\Gamma$ is arithmetic and nonprincipal, $T$ has an arithmetic model $A_{2}$ omitting $\Gamma$, thus $A_{1} \not A_{2}$. Also $\Gamma(\bar{c})$ has an arithmetic model $\left(A_{3}, \bar{c}\right)$ omitting $\Sigma(\bar{c}, \bar{y})$. Since $A_{3}$ realizes $\Gamma, A_{3} \neq A_{2}$. Also, since $A_{3}$ realizes $\Gamma$ it realizes every arithmetic type, so in particular it realizes $\Sigma(\bar{x}, \bar{y})$. But $\left(A_{3}, \bar{c}\right)$ does not realize $\Sigma(\bar{c}, \bar{y})$, so $A_{3}$ is not homogeneous. This shows $A_{3} \nRightarrow A_{1}$.

Finally, by [Rosenstein], $T$ has a weakly saturated countable model $A_{4}$ and a saturated countable model $A_{5}$. Since $T$ is not persistently arithmetic, neither $A_{4}$ nor $A_{5}$ is arithmetic. Thus

$$
A_{i} \equiv A_{j}, \quad i=1,2,3 ; j=4,5 .
$$

This completes the proof that $T$ must have at least rive countable models.

\section{REFERENCES}

M. Benda, Remarks on countable models, Fund. Math. 81 (1974), 107-119.

H. J. Keisler, Model theory for infinitary logic, North-Holland, Amsterdam, 1971.

T. S. Millar [1], Persistently finite theories with hyperarithmetic models, Trans. Amer. Math. Soc. (to appear).

T. S. Millar [2], Foundations of recursive model theory, Ann. Math. Logic 13 (1978), 45-72.

M. G. Peretyat'kin, Complete theories with a finite number of countable models, Algebra i Logika 12 (1973), 550-576.

J. G. Rosenstein, A note on a theorem of Vaught, J. Symbolic Logic 36 (1971), $439-440$.

R. L. Vaught, Denumerable models of complete theories, Infinitistic Methods (Proc. Sympos. Foundations of Math., Warsaw, 1959), Pergamon, Oxford; PWN, Warsaw, 1961, pp. 303-321.

Department of Mathematics, Monash University, Clayton, Australia

Department of Mathematics, University of Wisconsin, Madison, Wisconsin 53706 